\title{
HIPERGLICEMIA E MORBIMORTALIDADE EM CRIANÇAS GRAVES - ANÁLISE CRÍTICA BASEADA EM REVISÃO SISTEMÁTICA
}

\author{
Eduardo Mekitarian Filho ${ }^{*}$, Werther Brunow de Carvalho ${ }^{2}$, Eduardo Juan Troster ${ }^{3}$ \\ Trabalho realizado no Instituto da Criança Professor Pedro de Alcântara da Universidade de São Paulo - USP e Universidade Federal de São Paulo - UNIFESP, \\ S.Paulo, SP
}

\author{
*Correspondência: \\ Av. Damasceno Vieira, $n^{\circ} 820$ \\ - apto. 184 - Vila Mascote \\ São Paulo - SP \\ CEP: 04363-040 \\ Telefone: (11) 71527710 \\ emf2002@uol.com.br
}

\begin{abstract}
RESUMO
OвJEтıvo. Verificar se a hiperglicemia observada em pacientes de terapia intensiva pediátrica é fator de risco para o aumento da morbimortalidade e realizar uma análise crítica das pesquisas em pediatria e neonatologia.

Métodos. A técnica empregada foi uma revisão sistemática da literatura sobre hiperglicemia e terapia intensiva pediátrica. O levantamento bibliográfico foi realizado nos bancos de dados Medline, Lilacs, Cochrane Library e Embase, além da utilização de referências bibliográficas de textos escolhidos. Selecionados artigos em língua inglesa e espanhola sendo utilizados para pesquisa os termos hyperglycemia, intensive care units (pediatrics), hospitals, pediatrics e pediatric intensive care. Foram analisados estudos de coorte, retrospectivos e prospectivos. Os desfechos avaliados foram mortalidade durante internação em Unidade de Terapia Intensiva Pediátrica (UTIP); mortalidade durante internação hospitalar; tempo de internação em UTIP; tempo de internação hospitalar; mortalidade por doenças específicas; incidência de infecção; necessidade de ventilação pulmonar mecânica.

Resultados. Foram selecionados 79 artigos relacionados ao tema da pesquisa durante o período de estudo. Dentre estes, 15 (19\%) consistiam em estudos de coorte (Dois prospectivos e 13 retrospectivos), analisados separadamente.

Conclusão. A partir da análise dos estudos listados concluímos que há evidências de que a hiperglicemia, tanto isolada como persistente durante internação em UTIP, aumenta a morbimortalidade em crianças gravemente doentes, bem como seu tempo de internação hospitalar; entretanto, tais estudos apresentam problemas metodológicos como ausência de protocolos específicos de coleta glicêmica, desenho (maioria coortes retrospectivos) e falta da caracterização isolada da hiperglicemia como preditor de morbimortalidade, sendo necessários, portanto, novos estudos prospectivos.
\end{abstract}

Unitermos: Hiperglicemia. Morbidade. Mortalidade. Pediatria. Unidades de Terapia Intensiva.

\section{INTRODUÇÃO}

Diversas publicações dos últimos anos vêm demonstrando a importância das alterações endocrinológicas do paciente em terapia intensiva. As mesmas, além de muito frequentes, podem ser responsáveis por desfechos desfavoráveis às crianças doentes. não só pela frequência com a qual acomete os pacientes mas também pela hipótese cada vez mais considerada de haver relação estreita entre as mesmas e desfechos desfavoráveis aos doentes. A hiperglicemia está entre os mais importantes distúrbios e na população pediátrica, alvo desta revisão, estudos demonstram que tal alteração endocrinológica é altamente prevalente em crianças internadas em terapia intensiva.
As taxas de incidência são variáveis e altas, tanto para picos isolados quanto para hiperglicemia persistente durante a internação, podendo atingir até $90 \%$ de acordo com os estudos analisados quando o corte para hiperglicemia estabelecido é de $110 \mathrm{mg} / \mathrm{dL}^{1,2}$

Situações críticas de estresse orgânico, como ocorre nos pacientes internados em terapia intensiva, estão correlacionadas com a ativação do eixo hipotálamo-hipofisário e, assim, diretamente ligada à secreção de cortisol pelas glândulas adrenais. Tal mecanismo é fundamental para a manutenção da homeostase e da resposta orgânica ao estresse, e vem acompanhado pela secreção conjunta dos chamados hormônios contra-reguladores da insulina - adrenalina e noradrenalina, cortisol e hormônio do crescimento.

1. Especialista em Pediatria e Terapia Intensiva Pediátrica pela Sociedade Brasileira de Pediatria e Associação de Medicina Intensiva Brasileira, Médico Pediatra da Unidade de Terapia Intensiva do Hospital Santa Catarina e Pronto Socorro Infantil Sabará, São Paulo, SP

2. Livre-docente em Pediatria pela Universidade Federal de São Paulo, Escola Paulista de Medicina, Chefe da Unidade de Cuidados Intensivos Pediátricos do Hospital São Paulo, São Paulo, SP

3. Livre-Docente em Pediatria pela Universidade de São Paulo (USP), Chefe da Unidade de Terapia Intensiva Pediátrica do Hospital Israelita Albert Einstein, São Paulo, SP 
Ressaltam-se os efeitos pró-inflamatórios da glicose no estresse oxidativo celular através da liberação de radicais livres de oxigênio (peroxidação lipídica) ${ }^{3}$. A oxidação mitocondrial e o adequado funcionamento da respiração celular também são prejudicados em situação de hiperglicemia e foi demonstrado que em crianças com sepse e choque séptico meningocócico ${ }^{4}$ ocorre supressão da resposta inflamatória mediada por citocinas, devido à hiperglicemia e aos menores níveis circulantes de insulina em tal condição.

Tendo em vista tal substrato fisiológico, estudos recentes tentam demonstrar a correlação da hiperglicemia no paciente grave com possíveis piores desfechos durante sua internação em terapia intensiva. Na população adulta já é bem demonstrado que a hiperglicemia é responsável por maior morbimortalidade no paciente grave, bem como o fato de que o controle glicêmico estrito reduz a mortalidade de maneira significativa em pacientes cirúrgicos e a morbidade em pacientes clínicos ${ }^{5,6}$. Em pediatria, recentes estudos vêm crescentemente apontando a hiperglicemia como um fator contributivo para maior morbimortalidade durante internação em terapia intensiva. Segue, então, revisão crítica acerca das principais publicações até o momento que versam sobre $o$ assunto.

\section{Objetivo}

Verificar, pela análise sistemática das publicações disponíveis, se a hiperglicemia verificada em pacientes de terapia intensiva pediátrica é fator de risco para os seguintes desfechos: mortalidade durante a internação em Unidade De Terapia Intensiva Pediátrica (UTIP); mortalidade durante a internação hospitalar; tempo de internação em UTIP; tempo de internação hospitalar; mortalidade por doenças específicas; incidência de infecção; necessidade de ventilação pulmonar mecânica.

\section{MÉtodos}

O presente estudo consiste em uma revisão sistemática da literatura médica sobre o impacto da hiperglicemia em pacientes pediátricos gravemente doentes.

Identificação dos estudos. O levantamento bibliográfico foi realizado de maneira sistemática nos bancos de dados Medline (período de 1967 a junho de 2008), Lilacs (período de 1981 a junho de 2008), Cochrane Library (período de 1991 a junho de 2008) e Embase (período de 1980 a junho de 2008), além da utilização de referências bibliográficas de textos escolhidos. Foram analisados artigos em língua inglesa e espanhola; para a pesquisa foram utilizados os seguintes termos: hyperglycemia; intensive care units, pediatrics; hospitals, pediatrics e pediatric intensive care, sendo os três primeiros MeSh terms. A obtenção dos resultados ocorreu do cruzamento do MeSh hyperglycemia com os demais termos acima nas bases de dados citadas.

Critérios de seleção. Para a avaliação dos possíveis efeitos da hiperglicemia na evolução de crianças gravemente doentes foram considerados os seguintes critérios de seleção para os estudos:

Desenho dos estudos - estudos de coorte retrospectivo e prospectivo; população - de recém-nascidos até adolescentes com 18 anos de idade incompletos internados em unidade de terapia intensiva;
Critérios de exclusão - editoriais, relatos de casos, artigos que incluíram população adulta na análise, pacientes com diagnóstico prévio de diabetes melito, artigos de revisão não-sistemática, capítulos de livros, artigos cujo conteúdo não eram relacionados ao tema da pesquisa;

Definições - foram aceitos textos cujas metodologias definiam hiperglicemia como qualquer valor glicêmico aferido acima de 110 mg/dL, seja por medição de glicemia de ponta-de-dedo ou por dosagem laboratorial convencional;

Desfechos avaliados - mortalidade durante internação em unidade de terapia intensiva pediátrica (UTIP); mortalidade durante internação hospitalar; tempo de internação em UTIP; tempo de internação hospitalar; mortalidade por doenças específicas; incidência de infecção; necessidade de ventilação pulmonar mecânica.

\section{Resultados}

Foram selecionados 79 artigos relacionados ao tema do trabalho durante o período de estudo. Dentre estes, 14 textos (18\%) consistiam em revisões não-sistemáticas da literatura, 15 (19\%) consistiam em estudos de coorte (Dois prospectivos e 13 retrospectivos), quatro (5\%) eram editoriais e cartas a autores, $10(12,5 \%)$ incluíam pacientes com diabetes melito na análise dos dados, dois (2,5\%) continham adultos em seus dados, um $(1,3 \%)$ estava publicado em língua diferente dos critérios de inclusão do trabalho e 33 artigos encontrados (41,7\%) não tinham relação com o tema deste trabalho. Foram reservados para análise dentro dos critérios de inclusão os 15 estudos de coorte que procuraram estabelecer se a hiperglicemia era fator de risco para os desfechos listados em crianças internadas em unidades de terapia intensiva. Os resultados obtidos estão sintetizados na Tabela 1.

\section{Hiperglicemia e traumatismo crânioencefálico}

Os primeiros dados correlacionando hiperglicemia em crianças a piores desfechos foram publicados por Chiaretti $^{7}$ et al., em 1998. Os autores analisaram retrospectivamente os prontuários de 53 crianças admitidas em UTIP por traumatismo crânioencefálico (TCE) utilizando como desfecho a presença de sequelas neurológicas avaliadas pela escala de desfechos de Glasgow (GOS, Glasgow Outcome Scale) no período de um mês após a internação.

Os autores avaliaram 50 crianças internadas por traumatismo crânioencefálico (TCE) e fizeram medições seriadas da glicemia das mesmas durante a internação considerando o diagnóstico de hiperglicemia quando os valores ultrapassavam $150 \mathrm{mg} / \mathrm{dL}$. A hiperglicemia (valores acima de $150 \mathrm{mg} / \mathrm{dL}$ ) foi diagnosticada mais frequentemente em crianças com traumatismo crânioencefálico grave que aquelas com trauma leve ou moderado - em $87,5 \%$ das crianças com escore de coma de Glasgow (ECG) abaixo de 8 (média glicêmica de 237,8 $992 \mathrm{mg} / \mathrm{dL}$ ), em $60 \%$ dos pacientes com ECG entre 9 e 12 (média glicêmica de 178 \pm 78,7 $\mathrm{mg} / \mathrm{dL}$ ) e apenas em 25\% das crianças com ECG entre 13 e 15 (média de $131,5 \pm 39 \mathrm{mg} / \mathrm{dL}$ ). Neste mesmo texto foi feita a correlação entre os valores glicêmicos e o desfecho observado entre os pacientes - entre aqueles com bom desfecho neurológico, a média glicêmica foi aproximadamente $150 \mathrm{mg} / \mathrm{dL}$; dentre os com desfecho desfavorável (incluindo sequela neurológica 
grave e/ou óbito), as médias chegaram próximo a $250 \mathrm{mg} / \mathrm{dL}$. Os autores consideram na discussão os múltiplos fatores que podem estar envolvidos na gênese da hiperglicemia em crianças doentes, incluindo as com TCE, e sugerem com seus dados que pode haver relação entre o desfecho neurológico e as medidas glicêmicas iniciais e colocam a necessidade de maiores estudos para a confirmação de tal suposição.

No final do texto, os autores colocam a dúvida se havia correlação direta da hiperglicemia com desfecho ruim em crianças vítimas de TCE ou se o desenvolvimento de hiperglicemia durante a internação é o fator decisivo para tal desfecho, abrindo a lacuna para pesquisa.

Do mesmo modo, Cochran et al. ${ }^{8}$, em 2003, estudaram também vítimas de TCE. Os autores verificaram os registros hospitalares de crianças internadas no período de um ano, excluindo aquelas que não tinham ao menos uma medida glicêmica durante a internação. Assim, foram identificadas 293 crianças admitidas e excluídas 123 pelo motivo descrito. Foram utilizadas como variáveis para análise o ECG, o pH sanguíneo de admissão, a glicemia de admissão e o tempo de internação em UTIP.

Os pacientes que morreram tiveram níveis glicêmicos à admissão maiores que aqueles que sobreviveram (267 mg/ $\mathrm{dL}$ versus $135 \mathrm{mg} / \mathrm{dL}$ com $\mathrm{p}=0,000$ ). Neste estudo, ainda, a relação entre hiperglicemia e mortalidade foi demonstrada com uma razão de chances de 1,013 (intervalo de confiança de 95\% - 1,003-1,023); apesar da aparente pouca significância estatística, foi mais uma consistente avaliação dos possíveis efeitos deletérios da hiperglicemia em crianças vítimas de trauma. Os autores colocam este estudo como o primeiro a apresentar um limiar para hiperglicemia consistente com mortalidade pois, no trabalho, todas as crianças com glicemia inicial acima de 300 $\mathrm{mg} / \mathrm{dL}$ morreram. $\mathrm{O}$ estudo cita a hiperglicemia como um epifenômeno comumente presente em crianças graves e nas vítimas de TCE e aborda a dificuldade do estabelecimento de uma relação de causa-efeito entre hiperglicemia e desfecho neurológico desfavorável, colocando a necessidade de análises prospectivas detalhadas para a comprovação de tal relação.

\section{Hiperglicemia e pacientes em UTI pediátrica geral}

Srinivasan et al. ${ }^{1}$ analisaram, em 2004 , retrospectivamente, coorte de 179 crianças internadas em terapia intensiva, nãocirúrgicas, que estivessem em ventilação pulmonar mecânica e em uso de drogas vasoativas. Foi observada correlação direta entre picos glicêmicos e o desfecho principal avaliado que foi a mortalidade dos pacientes. Considerando-se as taxas de mortalidade, as menores médias glicêmicas estiveram presentes nos pacientes com menor mortalidade $(8,3 \%$ em pacientes com glicemias menores que $100 \mathrm{mg} / \mathrm{dL}$ em 24 horas; $12,5 \%$ de valores entre 100 e 140 mg/dL; 20\% entre 141 e 180 mg/dL e $28,5 \%$ de mortalidade naqueles com médias em 24 horas acima de $180 \mathrm{mg} / \mathrm{dL}$ ). A média glicêmica, o maior pico glicêmico e a duração da hiperglicemia foram variáveis de risco independentes para o aumento da mortalidade, todos com o nível de significância "p" menor que 0,01.

É importante salientar que neste estudo, tanto a regressão univariada quanto multivariada mostraram que a hiperglicemia é fator independente de risco para a mortalidade dos pacientes dentro da unidade de terapia intensiva pediátrica.

Faustino et al. ${ }^{2}$ analisaram três anos de internações em UTI Pediátrica de hospital terciário em coorte retrospectivo, utilizando dados de crianças que tinham ao menos uma aferição glicêmica durante a internação e que não fossem diabéticas. As variáveis independentes utilizadas para tal estudo foram a glicemia inicial, o maior valor glicêmico em 24 horas após a primeira coleta e o maior valor registrado em até 10 dias de internação após a primeira aferição. 0 desfecho primário avaliado foi a mortalidade intra-hospitalar, e o secundário o tempo de internação entre os pacientes sobreviventes, excluindo aqueles traqueostomizados. 0 aumento da mortalidade dos pacientes foi estatisticamente significativo naqueles que apresentaram picos glicêmicos em 24 horas da primeira coleta acima de $150 \mathrm{mg} / \mathrm{dL}$ (risco relativo 2,5; intervalo de confiança de 95\% - 1,26-4,93); tal achado teve ainda mais poder estatístico quando considerado o pico glicêmico máximo durante a internação acima de 120 mg/dL com risco relativo de 5,68 para um intervalo de confiança de $95 \%$ entre 1,38 e 23,47. No presente estudo não foi encontrada significância estatística em relação aos desfechos para a medida glicêmica admissional do paciente na UTI Pediátrica nem em relação ao tempo de internação. Os autores relatam dificuldades na análise dos dados por tratar-se de estudo retrospectivo com perda de dados e sem haver protocolo na unidade para definição da coleta das análises.

Em 2006, Wintergerst et al. publicaram interessante estudo ${ }^{9}$ analisando os efeitos tanto da hiperglicemia e hipoglicemia, mas também da variação glicêmica durante a internação, parâmetro este não avaliado anteriormente. 0 estudo foi realizado em UTI Pediátrica terciária com predomínio de pacientes cirúrgicos (cerca de 70\%) e o desfecho principal avaliado foi a mortalidade durante internação hospitalar. Os efeitos da hiperglicemia na mortalidade dos pacientes foi significativa, com $68 \%$ das mortes ocorrendo entre pacientes com níveis glicêmicos máximos superiores a 200 mg/dL com nível de significância "p" inferior a 0,001. A taxa de mortalidade foi de 5,7\% em níveis glicêmicos entre 110 e 150 mg/dL, 7,4\% entre 150 e 200 mg/dL e 9,9\% quando o nível glicêmico ultrapassou 200 mg/dL. Em contraste, pacientes com níveis glicêmicos normais tiveram mortalidade de apenas $1,5 \%$ como citado contando com apenas dois dos 50 óbitos observados em tal estudo. Mostra-se interessante, concluindo, o dado citado pelo autor que o parâmetro que mais obteve significância estatística relacionada ao aumento da mortalidade foi a variabilidade glicêmica. Nas crianças com variações grandes de glicemia, principalmente naquelas que experimentaram hipo e hiperglicemia a mortalidade foi ainda maior que aquelas que mostraram algum dos dois distúrbios isoladamente.

Analisando separadamente condições patológicas, Branco e colaboradores estudaram a glicemia e o risco de mortalidade em crianças com choque séptico ${ }^{10}$; desta vez, foi estudo de coorte prospectivo sendo todos pacientes refratários à fluidoterapia selecionados em um período de 32 meses para o estudo levando-se em consideração para relacionar com a mortalidade o pico glicêmico da internação. Nas crianças que morreram os picos glicêmicos foram significativamente maiores do que nas sobreviventes (262 $\pm 110 \mathrm{mg} / \mathrm{dL}$ contra $170 \pm 55 ; \mathrm{p}<0,01)$ e um pico glicêmico de $178 \mathrm{mg} / \mathrm{dL}$ teve sensibilidade de $71 \%$ 


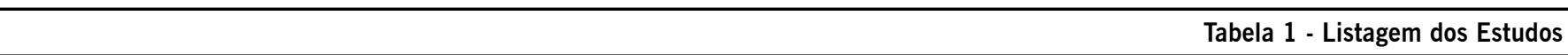

\section{Estudo}

Chiaretti et al. 1998

Gore et al. 2001

Cochran et al. 2002

Srinivasan et al. 2004

Hall et al. 2004*

Faustino et al. 2005

Yates et al. 2006

Wintergerst et al. 2006

Alaedeen et al. 2006*

Heimann et al. 2007*

Branco et al. 2007

Branco et al. 2007 (2)

Yung et al. 2008

Hirshberg et al. 2008

Falcao et al. 2008

\section{Desenho}

Coorte Retrospectiva

Coorte Retrospectiva

Coorte Retrospectiva

Coorte retrospectiva

Coorte retrospectiva

Coorte retrospectiva

Coorte retrospectiva

Coorte retrospectiva

Coorte retrospectiva

Coorte retrospectiva

Coorte prospectiva

Coorte retrospectiva

Coorte prospectiva

Coorte retrospectiva

Coorte retrospectiva

\section{No. Pacientes}

50

58

170

152

95

942 admissões

184

1094 admissões

50

\section{Métodos}

Análise de prontuários

Pacientes vítimas de TCE

Hiperglicemia - acima de $150 \mathrm{mg} / \mathrm{dL}$

Pacientes menores de 18 anos com SCQ > 60\% Definição arbitrária (controle glicêmico inadequado ruim quando mais de $40 \%$ das medidas acima de $140 \mathrm{mg} / \mathrm{dL}$ )

Pacientes vítimas de TCE

Pacientes em VPM e em uso de DVAs; definição > $126 \mathrm{mg} / \mathrm{dL}$

RNs com Enterocolite Necrosante ( > grau II)

Definição - > $120 \mathrm{mg} / \mathrm{dL}$ Todas admissões em UTIP

Pacientes maiores de 1 ano submetidos a bypass cardiopulmonar Definição - > $126 \mathrm{mg} / \mathrm{dL}$

Definição - > $110 \mathrm{mg} / \mathrm{dL}$

Todas admissões em UTIP

RNs menores de $1500 \mathrm{~g}$ em VPM; definição > 120 mg/dL e mortalidade

Prematuros $<1500 \mathrm{~g}$

3 coletas em 7 dias de vida

Definição - > $150 \mathrm{mg} / \mathrm{dL}$

Sepse e Choque Séptico Glicemia maior que $178 \mathrm{mg} / \mathrm{dL}$ teve S 71\% e E $72 \%$ para óbito

Bronquiolite menores de 2 anos Ventilação mecânica

Definição - > $110 \mathrm{mg} / \mathrm{dL}$ Relação com PELOD

Hiperglicemia $>150 \mathrm{mg} / \mathrm{dL}$ e hipoglicemia $<60 \mathrm{mg} / \mathrm{dL}$ Estudo da variabilidade glicêmica

Crianças em pós-operatório de cirurgia cardíaca

Legenda: TCE - Traumatismo Crânioencefálico; SCQ - Superfície Corpórea Queimada; VPM - Ventilação Pulmonar Mecânica; RNs - Recém-Nascidos; DVAs - Drogas Vasoativas; S - Sensibilidade; E - Especificidade; PELOD - Pediatric Logistic Organ Disfunction Score. *Estudos envolvendo apenas recém-nascidos. 


\section{Selecionados Para Revisão}

\section{Resultados}

A regressão linear entre hiperglicemia e ECG mostrou que 87,5\% das crianças com TCE grave tinham hiperglicemia $(p=0,0000)$

Maior incidência de alterações neurológicas

Crianças com controle glicêmico inadequado tiveram $27 \%$ de mortalidade contra $4 \%$ do grupo controle com $p<=0,05$ e foram submetidas a mais procedimentos cirúrgicos $(1,03+-0,09$ contra $0,9+-0,05), p<=0,05$

Maiores porcentagens de hemoculturas e culturas de feridas positivas, cirurgias, tempo de internação e mortalidade

Hiperglicemia foi fator isolado como preditor de mortalidade com razão de chances entre 1,003 - 1,023, IC 95\%

Maior incidência de alterações neurológicas e mortalidade

Duração da hiperglicemia esteve associados à maior mortalidade nas análises uni e multivariadas (razão de chances de 1,01 - 1,05 com p $<0,05$ )

Valores de razão de chances relacionados à hiperglicemia muito próximos da unidade

Maior mortalidade, picos e duração de hiperglicemia nos que morreram

RNs com maiores picos glicêmicos obtiveram maiores taxas de mortalidade após dez dias de admissão quando comparados aos normoglicêmicos

( $29 \%$ contra $2 \%, p=0,0009)$

Tempo de internação e mortalidade maiores

Picos glicêmicos acima de $150 \mathrm{mg} / \mathrm{dL}$ obtiveram razões de chances de 4,13 (1,83 - 9,32), IC 95\%, para mortalidade. Para tempo de internação, picos acima de $200 \mathrm{mg} / \mathrm{dL}$ predizeram maiores tempos quando comparados à crianças normoglicêmicas $(p<0,001)$

Tempo de internação e mortalidade maiores

Crianças com maiores incidências de hiperglicemia apresentaram à análise multivariada razão de chances de 1,48 para mortalidade $(p=0,002)$, de 1,59 para necessidade de OMEC ( $p=0,001)$ e de 1,48 para infecções nosocomiais $(p=0,002)$

Maiores incidências - Insuf. hepática e renal, diálise, Infecção, OMEC e eventos adversos de SNC

Picos glicêmicos acima de $150 \mathrm{mg} / \mathrm{dL}$ associaram-se à internação mais prolongada em UTIP e no hospital ( $<<0,001)$. Mortalidade em crianças com picos acima de $150 \mathrm{mg} / \mathrm{dL}$ foi de $17,3 \%$ contra $1,5 \%$ em normoglicêmicas $(p<0,001)$

Maior tempo de internação em UTI, hospitalar e mortalidade

Hiperglicemia correlacionou-se de maneira positiva com duração de nutrição parenteral $(p=0,005)$, VPM $(p=0,006)$ e hemoculturas positivas $(p=0,03)$

Maiores tempo de internação e mortalidade

RNs que morreram na primeira semana de vida tinham maiores picos glicêmicos $(p<0,0001)$ sem relação estatisticamente significativa com sepse, HPIV ou retinopatia da prematuridade

Maiores incidências de HPIV, retinopatia, sepse e hemoculturas positivas

Pacientes com picos glicêmicos acima de $178 \mathrm{mg} / \mathrm{dL}$ apresentaram mortalidade de $20 \%$ contra $8 \%$ dos demais, com $p<0,01$. A análise univariada mostrou razão de chances para mortalidade de 6,6 e a multivariada 6,1 para hiperglicemia com $p=0,005$

Maior mortalidade

Maior risco de morte nos maiores picos glicêmicos

Crianças com glicemia acima de $150 \mathrm{mg} / \mathrm{dL}$ tiveram maior tempo de VPM (5,5 - 9 dias versus 4,5 - 5 dias, $p<0,01)$, de internação em UTI (7 - 12 dias versus 4,5 - 8 dias, $p=0,01$ ) e maior necessidade de uso de inotrópicos ( $54 \%$ versus $14,8 \%, p=0,01$ )

Hiperglicemia não foi fator isolado para tempo de VPM e internação

Crianças hiperglicêmicas obtiveram razão de chances de 3,4 para PELOD > = $10(1,85$ - 6,46, $p<0,0001)$ e de 3,12 $(1,26$ - 7,7, p = 0,013) para mortalidade Maior escore PELOD e mortalidade Hiperglicemia - fator isolado

Crianças com picos glicêmicos acima de $150 \mathrm{mg} / \mathrm{dL}$ apresentaram razão de chances de 9,6 para mortalidade (IC 95\%, $p=0,004$ ) e de 2,0 para infecção nosocomial (IC 95\%, p =0,01)

Maiores mortalidade e infecção hospitalar em hiperglicêmicos e com variabilidade glicêmica

Maiores durações de hiperglicemia estiveram associadas à maiores taxas de infecção, insuficiência renal e hepática, eventis adversos de SNC, diálise e uso de OMEC (todos com $p<0,001$ ); maiores picos glicêmicos associados à maiores taxas de insuficiência renal e necessidade de OMEC Hiperglicemia foi fator isolado para maior morbimortalidade 
e especificidade de $72 \%$ para predizer o óbito em pacientes pediátricos, sendo o risco relativo de óbito neste grupo, cujo pico glicêmico excedeu $178 \mathrm{mg} / \mathrm{dL}$, de 2,59, variando o intervalo de confiança entre 1,37 e 4,88. No presente estudo, tanto na análise univariada como multivariada dos fatores de risco associados ao óbito das crianças, a hiperglicemia foi o único fator isoladamente envolvido para o nível de corte mencionado acima; nas duas análises, associou-se fortemente à mortalidade com nível de significância " $p$ " menor que 0,005. Ao final do texto, os autores mencionam a necessidade de se atingir níveis glicêmicos menores que $178 \mathrm{mg} / \mathrm{dL}$ caso seja comprovado que a insulinoterapia em crianças é eficaz para a diminuição da mortalidade, ao exemplo do observado em adultos, que utilizam níveis de corte glicêmicos menores como preditores de mortalidade e como gatilho para o início do tratamento.

Crianças com bronquiolite internadas em terapia intensiva pediátrica foram estudadas por Branco et al. ${ }^{11}$, em 2007, em uma coorte retrospectiva de 50 pacientes menores que dois anos e em ventilação pulmonar mecânica (VPM). Foram excluídas aquelas com evidências de infecção bacteriana complicando o quadro viral e aquelas em uso de corticoesteroides sistêmicos. Os desfechos previstos foram mortalidade durante internação, tempo de internação e duração de ventilação pulmonar mecânica.

Os níveis de corte para hiperglicemia foram de $110 \mathrm{mg} / \mathrm{dL}$ e $150 \mathrm{mg} / \mathrm{dL}$ e os desfechos avaliados foram tempo de ventilação pulmonar mecânica e tempo de internação em UTIP. Cerca de $98 \%$ das crianças admitidas tiveram picos glicêmicos acima de $110 \mathrm{mg} / \mathrm{dL}$. Nas crianças com glicemias acima de $150 \mathrm{mg} / \mathrm{dL}$ a duração da ventilação pulmonar mecânica foi maior que nas euglicêmicas (Seis dias com média entre 5,5-9 contra quatro dias com média entre 2,8-4,3; nível de significância " $p$ " < 0,01) bem como o tempo de internação em UTI (Nove dias com média entre 7-12 contra cinco dias com média entre 3,5-6,8; $p=0,01$ ); entretanto, a regressão múltipla dos dados mostrou que a hiperglicemia nãofoi fator independente responsável pelos desfechos buscados pelos autores. Ao final do texto, a falta de protocolo de coleta e as variáveis que podem estar relacionadas ao tempo de internação, como a disponibilidade de vagas por exemplo, são fatores que, segundo os autores, não permitem que seja estabelecida relação causal entre hiperglicemia e mortalidade.

Os dados de maior poder estatístico disponíveis foram recentemente obtidos por Hirshberg ${ }^{12}$ e colaboradores em 2008. Os mesmos estudaram 863 pacientes em coorte retrospectiva admitidas em UTIP e relacionou hipoglicemia ( $\leq 60 \mathrm{mg} / \mathrm{dL})$, hiperglicemia ( $\geq 150 \mathrm{mg} / \mathrm{dL}$ ) e variabilidade glicêmica (definida como presença de glicemias menores de $60 \mathrm{mg} / \mathrm{dL}$ e maiores que $150 \mathrm{mg} / \mathrm{dL}$ no mesmo paciente) à mortalidade, infecção hospitalar e tempo de internação hospitalar. Pacientes hiperglicêmicos tiveram razão de chances para mortalidade de 11,1 (variando entre 1,5 e 85,6 para intervalo de confiança de 95\%, $\mathrm{p}<0,001)$ comparados aos pacientes euglicêmicos e razão de 2,0 para infecção hospitalar (1-4,2 para IC 95\% com $p=$ $0,01)$; pacientes que experimentaram variabilidade glicêmica tiveram razão de chances de 63,6 (7,8-512,2 para IC 95\%, p $<0,001$ ) para mortalidade (confirmando os dados anteriormente publicados por Wintergerst e colaboradores ${ }^{9}$ ) e 4,9 para infecção hospitalar (1,9-12,7 para IC 95\%, $p=0,01)$. Os pacientes também ficaram por mais tempo internados, com significância estatística, que aqueles com níveis glicêmicos normais. Os autores citam ao final as limitações do estudo retrospectivo no que tange à ausência de protocolo de coleta e ao viés de amostragem, uma vez que crianças com medida anormal de glicemia acabam sempre tendo maior número de coletas de glicemia, aumentando de forma artificial o poder estatístico da hiperglicemia.

O segundo estudo de coorte prospectivo analisado foi recentemente publicado em 2008 por Yung et al. ${ }^{13}$ e incluíram 409 crianças internadas em UTIP e em ventilação pulmonar mecânica por mais de 12 horas. Yung et al. ${ }^{13}$ publicaram, em 2008, estudo de coorte prospectivo reunindo nove UTIs pediátricas na Austrália e Nova Zelândia e analisaram crianças em VPM por mais de 12 horas, excluindo as com diagnóstico de cetoacidose diabética, em ventilação domiciliar, com procedimentos cirúrgicos cardíacos e com ordens de não reanimação. Estabelecidos cortes para definição de hiperglicemia em 110 e 126 mg/dL e os desfechos avaliado foram a relação entre hiperglicemia e o escore pediátrico de disfunção orgânica (PELOD) e com a mortalidade. A análise univariada mostrou que a hiperglicemia esteve associada ao escore maior que $10 \mathrm{com}$ razão de chances de 3,4 (intervalo de confiança - IC - 95\% entre 1,85-6,46 e nível de significância "p" < 0,0001) e associada à mortalidade com razão de 3,12 (IC 95\% 1,26-7,7 com p < 0,001). Neste estudo, entretanto, não foi protocolada a coleta de glicemia, podendo levar a viés de seleção. Os autores comentam, a exemplo de outros estudos, que não foi possível estabelecer relação de causalidade, apenas associação entre hiperglicemia e as variáveis elencadas.

\section{Hiperglicemia e crianças em pós-operatório de cirurgia cardíaca}

Em crianças em pós-operatório de cirurgia cardíaca, Yates et al. publicaram, em 2006 ${ }^{14}$, a análise de que o período pósoperatório de 184 crianças era pior naqueles hiperglicêmicos, principalmente no que tange à duração da hiperglicemia. Pacientes com insuficiência renal aguda ou hepática, infecções ou eventos de sistema nervoso central tinham maior duração de hiperglicemia e morreram mais aqueles que tiveram maiores picos de glicemia. Este estudo, apesar de pioneiro neste grupo de crianças, incluiu apenas crianças menores de um ano e que submeteram-se exclusivamente a bypass cardiopulmonar, o que dificulta a extensão dos dados obtidos para a população pediátrica pós-operatória em geral.

Ratificando tal achado, recente estudo publicado em 2008 por Falcao et al. ${ }^{15}$ mostrou em analisaram coorte retrospectiva de 213 pacientes submetidos à cirurgia cardíaca (qualquer tipo de intervenção, paliativa ou corretiva) e encontraram altas incidências de hiperglicemia (chegando a 97\% das admissões). Os desfechos analisados foram a incidência de infecções,

insuficiências renal e hepática, eventos adversos de SNC, diálise e necessidade de OMEC.À regressão multivariada, a duração da hiperglicemia foi isoladamente associada à morbidade com razão de chances de 1,95 para nível de significância "p" menor que 0,001 e à mortalidade, com razão de chances de 1,41 para nível de significância " $p$ " igual a 0,03, mostrando que na população pediátrica pós-operatória em cirurgia cardíaca a hiperglicemia é,isoladamente, importante fator de risco adverso em relação à morbimortalidade. A duração da hiperglicemia, 
com $p<0,001$, só não foi relacionada de maneira significativa com a necessidade de diálise nestas crianças.

\section{Hiperglicemia em Crianças com Queimaduras Graves}

Continuando a análise em grupos específicos de pacientes, Gore e colaboradores publicaram em 2001 verificação de 58 pacientes menores de 18 anos admitidos em UTI para queimados $^{16}$ divididos em grupos nos quais a média glicêmica foi maior ou menor que $140 \mathrm{mg} / \mathrm{dL}$. A análise foi feita pela revisão de prontuários no período de 41 meses e procurou identificar todos os pacientes internados com superfície corpórea queimada maior que $60 \%$. Os desfechos a serem analisados foram a mortalidade hospitalar, tempo de internação, porcentagem de hemoculturas positivas e necessidade de procedimentos cirúrgicos. No grupo que manteve maiores médias glicêmicas, foi observada maior mortalidade (27\% contra apenas $4 \%, p<=0,05$ ) que aqueles que mantiveram, sem nenhum tipo de intervenção, glicemias menores. O tempo de internação hospitalar, quando considerados todos os pacientes elencados, foi maior naqueles com médias glicêmicas elevadas quando comparados aos com controle adequado (68 - 7 dias contra 51 - 6; $p=0,072$ ). Os autores comentam que, independentemente da relação causal, a hiperglicemia persistente está associada com piores desfechos aos pacientes grandes queimados, sugerindo que tal fato pode estar associado à redução da defesa antimicrobiana e imunodeficiência secundários à hiperglicemia, além do maior retardo na cicatrização de feridas. Entretanto, neste estudo não foram encontradas diferenças estatisticamente significantes entre taxas de infecção hospitalar e de ferida operatória entre crianças normoglicêmicas e hiperglicêmicas. Os autores postulam que, destemodo, qualquer fator que venha a contribuir para a pior evolução das queimaduras teria impacto negativo significativo no desfecho destes pacientes; entretanto, a hiperglicemia seguramente não é o único fator presente em um grande queimado que pode contribuir para tal aspecto.

\section{Hiperglicemia e Morbimortalidade no Período Neonatal}

Em relação aos pacientes recém-nascidos, Heimann et al. ${ }^{17}$ avaliaram de maneira retrospectiva os prontuários de 252 recémnascidos prematuros com peso de nascimento menor ou igual a 1500 g admitidos na unidade neonatal pelo período de quatro anos avaliaram os possíveis efeitos da hiperglicemia nesta faixa etária. Durante tal análise, foram considerados como desfechos a taxa de mortalidade e a incidência de sepse, hemorragia periintraventricular e retinopatia da prematuridade. Os recém-nascidos que morreram na primeira semana de vida tiveram níveis significativamente maiores que aqueles que sobreviveram com nível de significância " $p$ " < 0,0001. Os demais desfechos estudados não foram afetados pelos distúrbios glicêmicos. Neste estudo, não foi isolada a hiperglicemia como variável de risco, pois coincidentemente dentre os RNs que morreram, todos tinham idades gestacionais abaixo de 27 semanas e também estavam hiperglicêmicos.

Hall et al. publicaram análise retrospectiva, em $2004^{18}$, verificando 95 recém-nascidos com diagnóstico confirmado de enterocolite necrosante e analisaram os possíveis fatores de risco relacionados à mortalidade em terapia intensiva e ao tempo de internação. A análise foi realizada verificando-se os registros eletrônicos dos valores glicêmicos. Os desfechos principais avaliados foram o tempo de internação hospitalar e a taxa de mortalidade.

O autor encontrou que o pico glicêmico máximo acima de 200 mg/dL não se associou à maior mortalidade no cômputo geral porém foi estatisticamente significante a relação entre a mesma variável e a mortalidade tardia (Acima de 10 dias de admissão), com $p=0,0009$. Do mesmo modo, em regressão linear múltipla, dentre as variáveis elencadas (idade gestacional corrigida, peso de admissão, estadiamento da enterocolite, pico glicêmico máximo e necessidade de cirurgia) a única estatisticamente significativa para o maior tempo de internação, com $p$ $<0,0001$, foi o pico glicêmico.

Por fim, Alaedeen et al., em 2006, estudaram retrospectivamente $^{19}$ recém-nascidos admitidos com peso de nascimento também inferior a 1500 gramas com os seguintes critérios de inclusão: sepse (definida pelo autor como duas ou mais hemoculturas positivas),e hiperglicêmicos (acima de $120 \mathrm{mg} / \mathrm{dL}$ ), todos em nutrição parenteral total. Foram revistos os dados de prontuários dos RNs internados com tais critérios pelo período deum ano, totalizando 37 pacientes. Pacientes com níveis glicêmicos em média maiores ou iguais a $150 \mathrm{mg} / \mathrm{dL}$ tiveram duas vezes mais tempo de ventilação mecânica que aqueles com níveis menores que tal corte com $p=0,006$ e duas vezes mais tempo em nutrição parenteral prolongada ( $p=0,005)$. Os recémnascidos hiperglicêmicos também tiveram tempo de internação significativamente maior e maior mortalidade durante internação em UTI. Os autores comentam que a hiperglicemia associada à nutrição parenteral é fenômeno conhecido e de risco, novamente colocando em discussão a imunodeficiência transitória associada à hiperglicemia. É citado que neste estudo não foi possível avaliar se a hiperglicemia foi decorrente da infusão calórica excessiva ou se devido à ação dos hormônios contrareguladores, nem o efeito causal da mesma com a mortalidade.

\section{Discussão}

Sintetizando o que foi exposto neste trabalho, podemos concluir que a hiperglicemia é evento muito comum em pacientes de terapia intensiva (recém-nascidos, crianças e adultos);

que a hipoglicemia é menos freqüente, porém possivelmente mais ameaçadora como marcador prognóstico e que, de acordo com a maioria dos estudos mostrados, a hiperglicemia está associada também em crianças a desfechos clínicos desfavoráveis durante a internação hospitalar das mesmas.

É possível encontrar, na maioria dos estudos, diversas fontes de erros sistemáticos e aleatórios decorrentes do fato do delineamento de coorte retrospectivo.

Erros sistemáticos ocorrem pelo achado de resultados incorretos por causa de um viés, que pode ser de amostragem ou de seleção, diminuindo a exatidão dos dados obtidos. A falta de um protocolo definido de coleta de glicemia constitui-se viés de amostragem e pode ser observada em quase todos os estudos elencados. Assim, crianças com uma ou duas medidas glicêmicas normais tendem seguramente a terem menos coletas durante a internação. Por outro lado, o contrário faz com que crianças hiperglicêmicas tenham múltilpas medidas e, uma vez em que são na maioria dos casos mais graves que as normoglicêmicas, 
aumenta-se de maneira artificial o poder estatístico de correlação da hiperglicemia com qualquer desfecho.

Em alguns dos estudos, a amostra pequena de pacientes constitui-se em viés de seleção e impede, desta maneira, que os dados obtidos sejam extrapolados para a população pediátrica em geral. Em um ponto a maioria dos trabalhos é concordante, que é o pequeno número de critérios de exclusão (na maioria apenas crianças com diabetes mellitus), o que também é fonte de erro sistemático. Além disso, a característica retrospectiva impede a aleatorização da amostra. Tal fato também pode contribuir em viés de seleção.

Outro fator importante é a relação de causalidade entre hiperglicemia e os desfechos avaliados. Avaliando os critérios de Bradford-Hill para o estabelecimento de tal relação, podemos descrever:

- Força da associação - é bem descrita e adequadamente fundamentada na maioria dos estudos a associação entre hiperglicemia e morbimortalidade; sem demonstrar, entretanto, relação causal.

- Consistência dos achados em estudos de desenho semeIhante - também caracterizada em todos os trabalhos, principalmente pelos delineamentos de coorte retrospectivo, que mostram resultados semelhantes demonstrando associação estatística entre hiperglicemia e morbimortalidade.

- Causa precedendo efeito - é o fator principal a ser descrito pois é sabido que a hiperglicemia é um epifenômeno bem documentado em qualquer tipo de paciente grave pelos fatores descritos na introdução. Além disso, é multifatorial e o fato de ser sistematicamente observada hiperglicemia em crianças graves pode ser apenas uma coincidência neste sentido. Os estudos não discriminam se a hiperglicemia está diretamente envolvida na mortalidade ou se há apenas associação. A maioria dos textos exclui a possibilidade de se estabelecer relação causal entre as duas coisas.

- Plausibilidade biológica - existe de acordo com os estudos. De fato, há várias demonstrações dos efeitos deletérios da hiperglicemia in vivo e em modelos experimentais, de forma que é plausível estabelecer associação entre hiperglicemia e morbimortalidade.

- Efeito dose-resposta - em estudos que estabeleceram níveis de corte intermediários para hiperglicemia, sempre os valores mais elevados progressivamente tinham maiores significâncias estatísticas com a morbimortalidade.

Outro fator a ser considerado é a presença de variáveis de confusão, que podem estar ao mesmo tempo relacionadas à variável de predição (hiperglicemia) e de desfecho. Por exemplo, o uso de aminas vasoativas e corticosteroides pode estar presente em maior frequência em crianças graves, e também causar hiperglicemia. Tais variáveis não foram consideradas na maioria dos estudos. Demais variáveis a serem consideradas incluem o tipo de nutrição e as velocidades de infusão de glicose que certamente influem nos níveis glicêmicos.

Uma crítica a ser feita aos trabalhos citados reside no fato de a maioria dos mesmos ser retrospectiva e limitar-se a selecionar os sujeitos pela análise dos prontuários, verificar suas glicemias colhidas e cruzar os dados para verificar relação com a mortalidade. Com tais características, encontramos problemas como a perda ou falta de dados e a ausência de protocolos específicos de coleta de glicemia para os pacientes internados; sendo assim, encontramos em alguns estudos pacientes que tinham uma glicemia colhida e outros com diversas análises, independentemente da necessidade de coleta de cada grupo. Além disso, a maioria dos estudos não mostra se a glicemia sozinha foi capaz de discriminar como fator de risco isolado a mortalidade, uma vez que não foi descrito nos textos se coincidentemente os pacientes hiperglicêmicos eram mais graves previamente e, por isso, tiveram maiores taxas de mortalidade.

Sendo assim, parece ser importante o desenvolvimento de novos estudos de coorte prospectivos com uniformidade na coleta de glicemia das crianças internadas e associar, por exemplo, escores de gravidade aos valores encontrados e as condições de base das crianças, de modo a determinar se apenas os distúrbios da glicemia, independentemente do quadro clínico, podem ser responsáveis pelo aumento de mortalidade.

Os estudos apresentados mostram falhas metodológicas importantes, mas são uniformes em mostrar associação estatística entre hiperglicemia e maior morbimortalidade em crianças graves. Entretanto, nenhum deles é capaz de mostrar associação causal entre os dois fatores.

O estudo também verificou conjuntamente pacientes de UTI Pediátrica e Neonatal. Os resultados obtidos não podem ser extrapolados para as duas categorias de pacientes, tendo em vista as consideráveis diferenças fisiopatológicas e, consequentemente, nos efeitos da hiperglicemia em ambos.

Outro fator responsável neste sentido é a pequena proporção de estudos na faixa neonatal e apenas em situações específicas, dificultando ainda mais a avaliação.

Com dados mais consistentes disponíveis, poderíamos propor o controle glicêmico nas crianças de modo a reduzir a morbimortalidade das mesmas. O que não sabemos ainda é se em crianças o efeito da hiperglicemia é tão ruim quanto em adultos, levando em consideração que as crianças costumam apresentar menos comorbidades que os adultos; seus tecidos (como células beta, músculos e fígado) ainda não se submeteram ao estresse oxidativo e às variações de glicemia durante os anos. Do mesmo modo, há dificuldade na definição de euglicemia para pacientes pediátricos e teme-se o risco de agressão cerebral por hipoglicemia a uma criança com sistema nervoso ainda em formação. Por motivos como tais, o controle glicêmico ainda apresente muita resistência entre os intensivistas, guardadas as proporções dos pacientes pediátricos em relação aos adultos e a menor reserva glicêmica dos primeiros; de qualquer forma, apenas com a certeza do prejuízo de tal marcador para a saúde das crianças é que poderemos de maneira ativa intervir no prognóstico das mesmas.

\section{Conclusão}

A partir dos estudos apresentados, reunimos uma série de evidências em estudos pediátricos demostrando que a hiperglicemia está associada, em alguns casos de maneira significativa, a morbimortalidade de crianças gravemente doentes. Isto ocorre tanto no cômputo geral, analisando tempo de internação e taxa de mortalidade para qualquer admissão em UTIP, quanto em algumas condições específicas, como choque séptico e pósoperatório de cirurgia cardíaca. Tempo de internação em UTIP e tempo de internação hospitalar total também se correlacionaram 
aos maiores picos glicêmicos. Apenas um estudo correlacionou aumento do tempo de ventilação pulmonar mecânica e em recém-nascidos e, nesta mesma população, uma pesquisa obteve maiores índices de infecção em crianças hiperglicêmicas e na população pediátrica geral uma pesquisa descrita também citou maiores incidências de infecção hospitalar em crianças hiperglicêmicas e submetidas a grandes variabilidades glicêmicas. Não é possível pelos motivos descritos, estabelecer relação de causalidade entre hiperglicemia e morbimortalidade, apenas associação estatística. Apesar das limitações metodológicas já descritas acima, parece-nos importante a discussão cada vez mais aprofundada do assunto, de modo a entendermos melhor o quanto é necessária a intervenção para manter a glicemia estritamente dentro dos valores considerados normais.

Conflito de interesse: não há

\section{SUMmary}

Hyperglycemia, Morbidity and Mortality in Critically Ill Children - Critical Analysis Based on a Systematic Review

OBJECTIVE. This article focused on verifying if hyperg/ycemia in critically ill pediatric patients is a risk factor for increased morbidity and mortality and carried out a critical analysis of the articles in pediatrics and neonatology.

METHODS. A systematic review of literature was performed using Medline, Cochrane, Lilacs and Embase databases and references of articles. Articles written in Portuguese, English and Spanish were selected and the terms used in the search were hyperglycemia, intensive care units (pediatrics), hospitals, pediatrics and pediatric intensive care. Cohort studies, retrospective and prospective, were selected for analysis. The outcomes evaluated were mortality during pediatric intensive care unit (PICU) stay, mortality during hospital stay, length-ofstay in the PICU, mortality due to specific diseases, and risk of infection and time of mechanical ventilation.

RESULTS. During the study period 79 articles related to hyperglycemia in critically ill pediatric patients were selected; 15 (19\%) were cohort studies (2 prospective and 13 retrospective) that were analyzed separately.

CONCLUSION. Analysis of these cohort studies supported the conclusion that hyperglycemia, isolated or persistent during stay in PICU, increases morbidity, mortality and length-of-stay in PICU of critically ill children. However, these studies disclosed methodological issues such as lack of protocols for glucose measurement, design (most of them retrospective cohorts) and many articles did not confirm hyperglycemia as a single predictor of morbidity and mortality in pediatrics; therefore further prospective studies are necessary. [Rev Assoc Med Bras 2009; 55(4): 475-83]

KEYwords: Hyperglycemia. Intensive care units. Morbidity. Mortality. pediatrics.

\section{REFERÊNCIAS}

1. Srinivasan V, Spinella PC, Drott HR, Roth CL, Helfaer MA, Nadkarni V. Association of timing, duration, and intensity of hyperglycemia with intensive care unit mortality in critically ill children. Pediatr Crit Care Med. 2004; 5:329-36.

2. Faustino EV, Apkon M. Persistent hyperglycemia in critically III children. J Pediatr. 2005;146:30-4.

3. Raghavan M, Marik PE. Stress hyperglycemia and adrenal insufficiency in the critically III. Semin Respirat Crit Care Med. 2006;27:274-85.

4. Van Waardenburg DA, Jansen TC, Vos GD, Buurman WA. Hyperglycemia in children with Meningococcal sepsis and septic shock: the relation between plasma levels of insulin and Inflammatory Mediators. J Clin Endocrinol Metab. 2006;91:3916-21.

5. Van den Berghe G, Wouters P, Weekers F, Verwaest C, Bruyninckx F, Schetz $\mathrm{M}$, et al. Intensive insulin therapy in critically ill patients. N Engl J Med. 2001;345:1359-67.

6. Van den Berghe G, Wilmes A, Hermans G, Meersseman W, Wouters PJ, Milants I, et al. Intensive insulin therapy in the Medical ICU. N Engl J Med. 2006; 354:449-61.

7. Chiaretti A, De Benedictis R, Langer A, Di Rocco C, Bizzarri C, lannelli A, et al. Prognostic implications of hyperglycaemia in paediatric head injury. Child's Nerv Syst. 1998; 14:455-9.

8. Cochran A, Scaife ER, Hansen KW, Downey EO. Hyperglycemia and outcomes from pediatric traumatic brain injury. J Trauma. 2003;55:1035-8.

9. Wintergerst KA, Buckingham B, Gandrud L, Wong BJ, Kache S, Wilson DM. Association of hypoglycemia, hyperglycemia, and glucose variability with morbidity and death in the pediatric intensive care unit. Pediatrics. 2006;118:173-9.

10. Branco RG, Garcia PCR, Piva JP, Casartelli CH, Seibel V, Tasker RC. Glucose level and risk of mortality in pediatric septic shock. Pediatr Crit Care Med. 2005;6:470-472.

11. Branco RG, Tasker RC. Glycemic level in mechanically ventilated children with bronchiolitis. Pediatr Crit Care Med. 2007;8:546-50.

12. Hirshberg E, Larsen G, Van Duker H. Alterations in glucose homeostasis in the pediatric intensive care unit: Hyperglycemia and glucose variability are associated with increased mortality and morbidity. Pediatr Crit Care Med. 2008;9:361-6.

13. Yung M, Wilkins B, Norton L, Slater A. Glucose control, organ failure, and mortality in pediatric intensive care. Pediatr Crit Care Med. 2008;9:147-52.

14. Yates AR, Dyke PC 2nd, Taeed R, Hoffman TM, Hayes J, Feltes TF, et al. Hyperglycemia is a marker for poor outcome in the postoperative pediatric cardiac patient. Pediatr Crit Care Med. 2006;7:351-5.

15. Falcao G, Ulate K, Kouzekanani K, Bielefeld MR, Morales JM, Rotta AT. Impact of postoperative hyperglycemia following surgical repair of congenital cardiac defects. Pediatr Cardiol. 2008;3:628-36.

16. Gore DC, Chinkes D, Heggers J, Herndon DN, Wolf SE, Desai M. Association of Hyperglycemia with Increased Mortality after Severe Burn Injury. J Trauma. 2001;51:540-4.

17. Heimann K, Peschgens T, Kwiecien R, Stanzel S, Hoernchen H, Merz U. Are recurrent hyperglycemic episodes and median blood glucose level a prognostic factor for increased morbidity and mortality in premature infants. J Perinat Med. 2007;35:245-8.

18. Hall NJ, Peters M, Eaton S, Pierro A. Hyperglycemia is associated with increased morbidity and mortality rates in neonates with necrotizing enterocolitis. J Pediatr Surg. 2004;39:898-901.

19. Alaedeen DI, Walsh MC, Chwals WJ. Total parenteral nutrition-associated hyperglycemia correlates with prolonged mechanical ventilation and hospital stay in septic infants. J Pediatr Surg. 2006;41:239-44.

Artigo recebido: 05/06/08 Aceito para publicação: 07/03/09 